\title{
MAPEAMENTO DE RECIFES SUBMERSOS NA COSTA DO RIO GRANDE DO NORTE, NE BRASIL: MACAU A MARACAJAU
}

\author{
Claude Luis Aguilar Santos ${ }^{1,2}$, Helenice Vital ${ }^{1,3}$, Venerando Eustáquio Amaro ${ }^{1,3}$ e \\ Ruy Kenji Papa de Kikuchi ${ }^{4}$
}

Recebido em 6 maio, 2005 / Aceito em 11 janeiro, 2006

Received on May 6, 2005 / Accepted on January 11, 2006

\begin{abstract}
Studies on the Brazilian continental shelf were developed between Touros and Macau-RN (NE Brazil) with the aim to mapping carbonate buildups, and especially, reef ecosystems. Remote sensing, submarine movies, bathymetric survey and diving were the methods used. This paper will focus on three main aspects of the Rio Grande do Norte reefs: 1) characterization of the coral fauna; 2) the classification and 3) the distribution of the main northriograndense carbonate buildups.

Reefs environments are found predominantly on the inner shelf adjacent to Touros, at depths shallower than $10 \mathrm{~m}$. These reefs generally show NW-SE orientation parallel to the coastline and constitute groupings of knolls and patch reefs. A prominent carbonate buildup, where the corals are almost absents and algae incrustation is thin, occurs in average depths of $25 \mathrm{~m}$, along the northern part of Rio Grande do Norte State and is classified as sandstone bank. Others sandstone banks, with smaller lengths and heights were mapped around $10 \mathrm{~m}$ depth. They should be related to an ancient coastline; however the corresponding lithostratigraphic unit was not defined. This work is a contribution to the mapping of coral reef, in particular, and carbonate buildups, in general, on the Rio Grande do Norte coast.
\end{abstract}

Keywords: reefs, Rio Grande do Norte, remote sensing, continental shelf.

RESUMO. Estudos na plataforma continental brasileira entre Touros e Macau-RN (NE do Brasil) foram realizados com o objetivo de mapear construções carbonáticas, inorgânicas e recifais, utilizando técnicas de sensoriamento remoto, filmagem submarina, levantamento batimétrico e mergulho in situ. Três aspectos são abordados: 1) caracterização da fauna de corais; 2) classificação e 3) distribuição das principais construções carbonáticas desta costa norteriograndense. Os resultados obtidos mostram que os ambientes recifais ocorrem predominantemente na plataforma interna da região de Touros (recifes de Sioba, Cação, Rio do Fogo e Maracajau), litoral oriental. Estes recifes se aglomeram em conjuntos de cômoros (kno//) e canteiros (patch) e essas aglomerações tomam geralmente orientação NW-SE, paralelos à linha de costa. Construções carbonáticas inorgânicas, onde a presença de corais é inexpressiva, ocorrem a uma profundidade média de $25 \mathrm{~m}$ (entre as isobátas de 20 e 30 m), ao longo de toda a área estudada, sendo classificados como bancos areníticos. Outras construções inorgânicas, embora de menor extensão e menor altura, foram mapeadas a profundidades em torno de $10 \mathrm{~m}$. Podem ser relacionadas a uma linha de costa pretérita. Entretanto, a unidade litoestratigráfica correspondente esses bancos não foi definida. 0 mapeamento das construções carbonáticas (e em especial dos recifes) desenvolvido neste trabalho se constitui em uma contribuição ao levantamento de dados sobre ocorrência de corais na costa do Rio Grande do Norte.

Palavras-chave: recifes, Rio Grande do Norte, sensoriamento remoto, plataforma.

Universidade Federal do Rio Grande do Norte - UFRN, Cx. Postal 1596, 59072-970, Natal, RN, Brasil. Telefone: ++55 (84) 215-3727 R-13, 215-3808 R-213; Fax: ++55 (84) 215-3683 R-11 - E-mails: claude@ufrnet.br / helenice@geologia.ufrn.br / amaro@geologia.ufrn.br

1 Programa de Pós-graduação em Geodinâmica e Geofísica - PPGG.

${ }^{2}$ Museu Câmara Cascudo/UFRN.

${ }^{3}$ Departamento de Geologia/UFRN.

${ }^{4}$ Universidade Federal da Bahia - UFBA. Centro de Pesquisa em Geofísica e Geologia - CPGG. E-mail: rkikuchi@cpgg.ufba.br 


\section{INTRODUÇÃo}

0 nível do mar, referencial utilizado na maioria das vezes para avaliação das variações topográficas e batimétricas, sofreu variações significativas ao longo do tempo geológico exercendo papel de destaque na evolução das paleolinhas de costa e áreas vizinhas. Os recifes afiguram-se como importante registro dessas variações eustáticas ocorridas durante 0 Quaternário e, em especial, no Holoceno (Chappell, 1983; Davies \& Montaggioni, 1985; Hopley, 1986; Kikuchi \& Leão, 1998), como edificações biológicas cuja evolução está condicionada pela variação vertical do nível do mar. Bancos de arenitos de praia, podendo ser também recobertos superficialmente por uma cobertura carbonática orgânica, tal como descrito por Nolasco \& Leão (1986), são construções que evidenciam tanto 0 deslocamento das linhas de costa em relação ao continente (Davies \& Kinsey, 1973; Oliveira et al., 1990) como variações verticais desse nível. Em ambos os casos ficam evidentes as alterações ao longo do tempo da situação geográfica das condições ambientais (fatores limitantes) determinantes à gênese dos recifes.

A presença de construções carbonáticas, e em especial de recifes ao longo da costa brasileira é uma característica marcante. 0 interesse pela ocorrência de recifes, justificado pelo risco que representa à navegabilidade de embarcações ou pelo interesse no ecossistema (Hartt, 1870), evoluiu na medida em que avançaram os conhecimentos sobre sua origem, estrutura e importância econômica e ambiental, bem como sua relação com a dinâmica costeira.

As construções carbonáticas podem ser classificadas, de acordo com sua origem, em (i) bancos carbonáticos inorgânicos - formados sob condições ambientais características da linha de costa, conhecidos como arenito de praia ou rocha praial (beachrocks), testemunhando antigas linhas de costa, com orientações Iongitudinais muito próximas entre si e em relação à atual linha de costa; e (ii) recifes - estruturas rochosas, rígidas, resistentes à ação mecânica das ondas e de correntes marinhas, edificados pela ação de organismos marinhos (animais e vegetais), capazes de segregar esqueletos a base de carbonato de cálcio, usando como substrato rochas sedimentares, ígneas ou metamórficas disponíveis a uma profundidade adequada para 0 seu estabelecimento e desenvolvimento (Leão, 1994). Os recifes podem se desenvolver sobre substratos os mais diversos: recifes pleistocênicos (Leão \& Lima, 1982), rochas sedimentares das bacias marginais cretácicas (Araújo et al., 1984), embasamento cristalino ou arenitos de praia (Nolasco \& Leão, 1986), ou ainda rochas vulcânicas (Kikuchi \& Leão, 1997). Em praticamente todas as situações citadas, a espessura máxima de carbonato de cálcio depositado por corais e algas é de cerca de $12 \mathrm{~m}$ (Leão et al., 2003). Além disso, são classificados de acordo com a sua distância da costa, dimensões (comprimento $\times$ altura) e relação com a evolução costeira (Leão et al., 2003). Assim, pode ocorrer de uma construção carbonática inorgânica, como os beachrocks, virem a se tornar substrato de recifes. Estes, quando encontrados num estágio inicial de desenvolvimento (espessura de carbonato de cálcio orgânico - algas e corais principalmente - inferiores a $0,5 \mathrm{~m}$ ) foram chamados de recifes superficiais por Nolasco \& Leão (1986). Neste caso, os recifes são alongados e paralelos à linha de costa, e em algumas situações, podem ser confundidos com os arenitos de praia (seu substrato), e vice-versa.

Branner (1904) já chamava atenção ao fato de que boa parte das construções carbonáticas localizadas entre 0 Maranhão e a Bahia é não orgânica. Entretanto o interesse acerca dos recifes da costa brasileira tem se expandido nas últimas décadas, devido ao aumento no número de pesquisadores trabalhando neste campo de investigação, e ao incremento de degradação de áreas recifais (Leão et al., 2003). Apesar do incremento nas informações científicas acerca dos recifes do Brasil, o conhecimento sobre suas condições atuais é ainda escasso, e em algumas áreas são virtualmente desconhecidas.

No Rio Grande do Norte, diversos trabalhos vêm indicando idades holocênicas e pleistocênicas para os beachrocks, situados na extensão da faixa costeira adjacente à área submersa selecionada para estudo, e cujas posições atuais em relação ao nível do mar indicam variações do nível eustático (Barreto et al., 2002; Caldas, 2002; Stattegger et al., 2006). Entretanto, estudos sistemáticos visando à identificação e mapeamentos destas construções carbonáticas (inorgânicas e/ou recifais) em áreas da plataforma são restritos à região de Touros e baseados principalmente em sensores remotos (Vianna et al., 1991,1993; Lima \& Amaral, 2001; Amaral, 2002; Testa, 1997; Testa \& Bosence, 1998, 1999). Estes autores reconhecem a importância de associar a investigação de sensores remotos a outros estudos sistemáticos.

Assim este trabalho tem como objetivo principal mapear as edificações carbonáticas presentes na plataforma continental brasileira, entre Touros e Macau-RN (Fig. 1), utilizando técnicas de sensoriamento remoto, filmagem submarina e mergulho in situ. Nesse quadro de particularidades regionais o estágio de conhecimento é ainda incipiente sobre os recifes da costa do RN.

A importância deste tipo de estudo pode ser avaliada de forma sumária ao considerarmos que, embora representem apenas 0,2\% de toda a área oceânica do planeta, são áreas de alta diversidade biológica e produtividade. Podem ser nestes termos, equiparáveis somente às florestas tropicais (Roger, 1974; entre outros). 


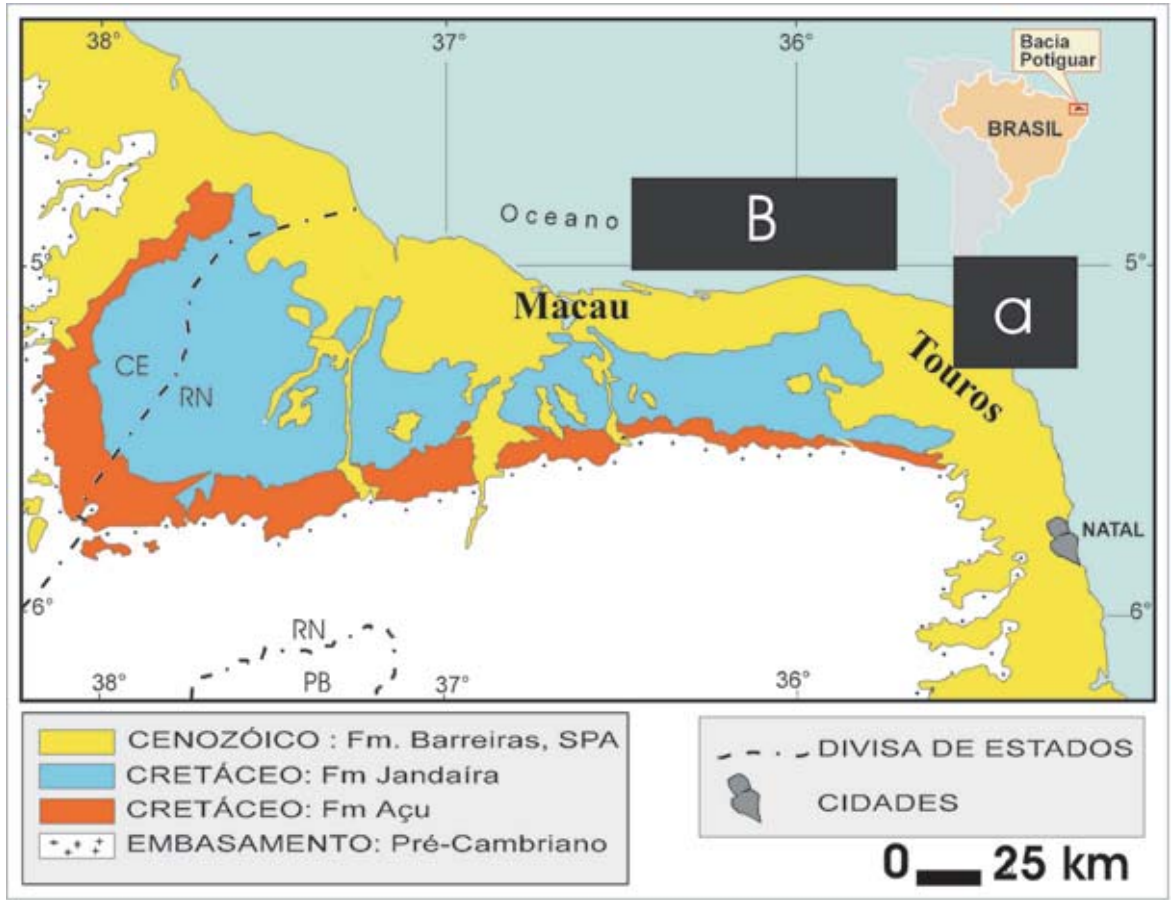

Figura 1 - Mapa geológico simplificado da Bacia Potiguar (RN) com localização da área mapeada em preto. SPA = sedimentos de praia, dunas e aluvião.

\section{ASPECTOS GEOAMBIENTAIS}

A área de estudo engloba partes do litoral oriental (Touros) e setentrional do Rio Grande do Norte (Macau). A região de Touros, no litoral oriental (área a, Fig. 1) está sob o regime climático tipo Af, "tropical úmido" segundo Köppen, com precipitação pluviométrica anual em torno de $1500 \mathrm{~mm}$ por ano, com temperatura mínima em torno de $20^{\circ} \mathrm{C}$, média $27^{\circ} \mathrm{C}$ e máxima de $32^{\circ} \mathrm{C}$; com direção de ventos predominantes de sudeste (Nimer, 1989). Atualmente constitui-se em Área de Proteção Ambiental - APA dos Recifes de Corais, criada pelo Governo do Estado do Rio Grande do Norte, através do Decreto n 15.476, de 6 de junho de 2001. Já a região de Macau, no litoral setentrional (área $\mathbf{b}$, Fig. 1), está sujeita à influência climática que domina na porção setentrional do estado nas regiões contíguas continentais e costeiras. Clima Bsw'h, "semi-árido moderado" segundo a classificação de Köppen, com precipitação pluviométrica compatível com um regime tropical de zona equatorial. Temperatura mínima na primavera, e máxima no outono. Médias mensais oscilando de $26^{\circ}$ a $27^{\circ} \mathrm{C}$, máxima absoluta de $38^{\circ} \mathrm{C}$ (Nimer, 1989).

De modo geral, a presença de recifes nestas áreas viabilizou 0 estabelecimento de um conjunto de instalações e equipamentos empresariais, e outras atividades menores, relacionados com a extração de petróleo, exploração de calcários, areias, argilas, diatomita, submetendo a área a impactos diretos e indiretos, cujos efeitos se fazem sentir visivelmente através de drásticas alterações ambientais na região ou através de monitoramento. 0 turismo é também uma atividade econômica em expansão nessas áreas.

A área mapeada compreende a plataforma continental brasileira entre Touros e Macau-RN (Fig. 1), onde perfilam as estruturas recifais, popularmente conhecidas e assinaladas nas cartas náuticas da DHN como: Baixos de Maracajau, Rio do Fogo, Cação, Sioba; Urcas do Tubarão, Minhoto, Conceição; Risca do Zumbi. Geologicamente, encontra-se situada no contexto geológico da Bacia Potiguar (Fig. 1), extremo Nordeste do Brasil. Esta bacia abrange uma área de $48.000 \mathrm{~km}^{2}$, sendo $21.500 \mathrm{~km}^{2}$ em terra e $26.500 \mathrm{~km}^{2}$ até a isóbata de $-2000 \mathrm{~m}$, estando implantada na Província Borborema, de Almeida et al. (1977). À semelhança de outras, como as bacias do Recôncavo, Tucano e Jatobá, Rio do Peixe e Sergipe - Alagoas, a Bacia Potiguar faz parte do Sistema de Rifte do Nordeste Brasileiro (Matos, 1992).

A partir dos estudos de diversos autores (Bertani et al., 1987; Cremonini et al., 1996; entre outros), pode ser traçada uma configuração geométrica da estrutura da bacia, a qual seria constituída por um conjunto de graben's assimétricos (Apodi, Umbuzeiro, Guamaré e Boa Vista) de direção NE, levemente oblíquos 
aos principais lineamentos do embasamento cristalino a sul da bacia (Fig. 2). Altos do embasamento separam os principais graben's da bacia. Esses altos consistem de cristas alongadas formadas por gnaisses, migmatitos ou xistos, soerguidos por falhas normais (Macau, Serra do Carmo e Quixaba). A Bacia Potiguar está limitada a oeste, com a Bacia do Ceará, pelo Alto de Fortaleza, e a leste, com a Bacia Pernambuco - Paraíba, pelo Alto de Touros (Araripe \& Feijó, 1994). Ao sul, o limite é constituído pela discordância com 0 embasamento cristalino, enquanto a norte e leste, pela cota batimétrica de $-2000 \mathrm{~m}$.

As condições geoambientais (clima, relevo, hidrografia, salinidade, recursos naturais) condicionaram em vários aspectos, 0 processo de colonização e ocupação humana da região. Várias dessas condições são apontadas como adequadas para 0 estabelecimento de empresas como as mencionadas anteriormente, $\mathrm{e}$ cujo retorno exige cuidados voltados a algumas conseqüências já bem conhecidas, que afetam as condições sócio-econômicas das comunidades, tais como alterações na qualidade e produtividade do pescado na região, mudanças na composição da fauna e flora, alterações do relevo com modificações na direção dos fluxos das vertentes, barramento do fluxo dos rios e mudanças nas variações de salinidade, alterações dos hábitos e condições de subsistência dos habitantes locais.

\section{MÉTODOS}

A metodologia utilizada constou inicialmente de levantamento de dados sobre a área selecionada para estudo, e pertinentes a outras áreas similares, nacionais ou internacionais, seguida de elaboração de mapa base de ocorrências de construções carbonáticas, e em especial de recifes na plataforma do Rio Grande do Norte, através de imagens de satélite e dados batimétricos. As imagens digitais do Landsat 5-TM de 02 de agosto 1998 (condições de maré de baixamar) e Landsat 7-ETM+ de 12 de junho 2000 (condições de maré de baixamar), foram utilizadas nas bandas do visível-infravermelho (bandas multiespectrais de 1-5 e 7). 0 software ER-Mapper v. 6.2 foi empregado para 0 processamento digital de imagens e 0 software ArcView para integração e elaboração dos mapas temáticos. 0 processamento envolveu 0 georreferenciamento e 0 registro das imagens digitais, 0 cálculo da estatística das imagens, a integração das multibandas por meio das composições coloridas RGB (Sistema de Cores Red-Green-Blue) e RGBI (Sistema de Cores Red-GreenBlue-Intensity), imagens resultantes de razões de bandas, além da utilização de processos de filtragem e análise das principais componentes (PCs).
A partir do mapa base gerado (Fig. 3), foi realizado merguIho autônomo para reconhecimento e comprovação das feições mapeadas por satélite e para filmagem do fundo marinho. Foram realizados mergulhos em duas estações na Região de Touros (Rio do Fogo e Maracajau). Em cada uma delas foram realizados 12 levantamentos com vídeo. No litoral setentrional, as construções foram investigadas em duas estações de mergulho. Nestas estações, foram coletadas amostras superficiais da construção carbonática e realizada a sua descrição petrográfica. Coleta de sedimentos superficiais com draga pontual tipo van-veene levantamento batimétrico foram necessários para calibração das feições observadas. 0 ecobatímetro utilizado foi um Hydrotac da Odom Hydrographic System, com GPS acoplado, resolução de 0,01 m e operando em freqüência de $200 \mathrm{kHz}$. Para o levantamento batimétrico foram realizados perfis transversais à costa a cada $1 \mathrm{~km}$.

Os recifes mapeados foram classificados de acordo com Leão (1994) e Leão et al. (2003).

\section{RESULTADOS E DISCUSSÕES}

0 mapa apresentado na Figura 3, obtido a partir da integração do processamento das imagens de satélite e estudos in situ, mostra a distribuição dos recifes e construções carbonáticas inorgânicas presentes na área estudada.

Os resultados obtidos mostram que as feições predominantes na plataforma interna da região de Touros (recifes de Sioba, Cação, Rio do Fogo e Maracajau), litoral oriental (Figs. 1a e Fig. 3a), estão representadas por um complexo de recifes. Laborel $(1967,1969)$ descreve-os como grupo de recifes de forma oval, localizados a alguns quilômetros para fora da costa, constituindo-se em estruturas simples, formadas em geral por numerosos pináculos num fundo arenoso raso e número reduzido de espécies, onde Siderastrea stellata (Verrill, 1868), principal coral formador dos recifes nesta região, com 80\%, ocorrem no platô destes recifes, enquanto algas calcárias Melobesiaea e gastrópodes vermetídeos formam uma crista no lado voltado para o mar, acompanhada por uma zona dominada pelo hidróide calcário Millepora alcicornis (Linné, 1758) com a espécie de coral Mussismilia hartii (Verrill, 1868) na escarpa da sua parede lateral. Maida \& Ferreira (2004) relatam adicionalmente a presença de Favia gravida (Verrill, 1886) no platô dos recifes e Monstastrea cavernosa em maiores profundidades.

A composição colorida em 4R3G2B foi a que melhor ressaltou os recifes de coral (Fig. 4a). Encontram-se a profundidades menores que 10 e a uma distância de 5 a 7 km da costa. Estes re- 


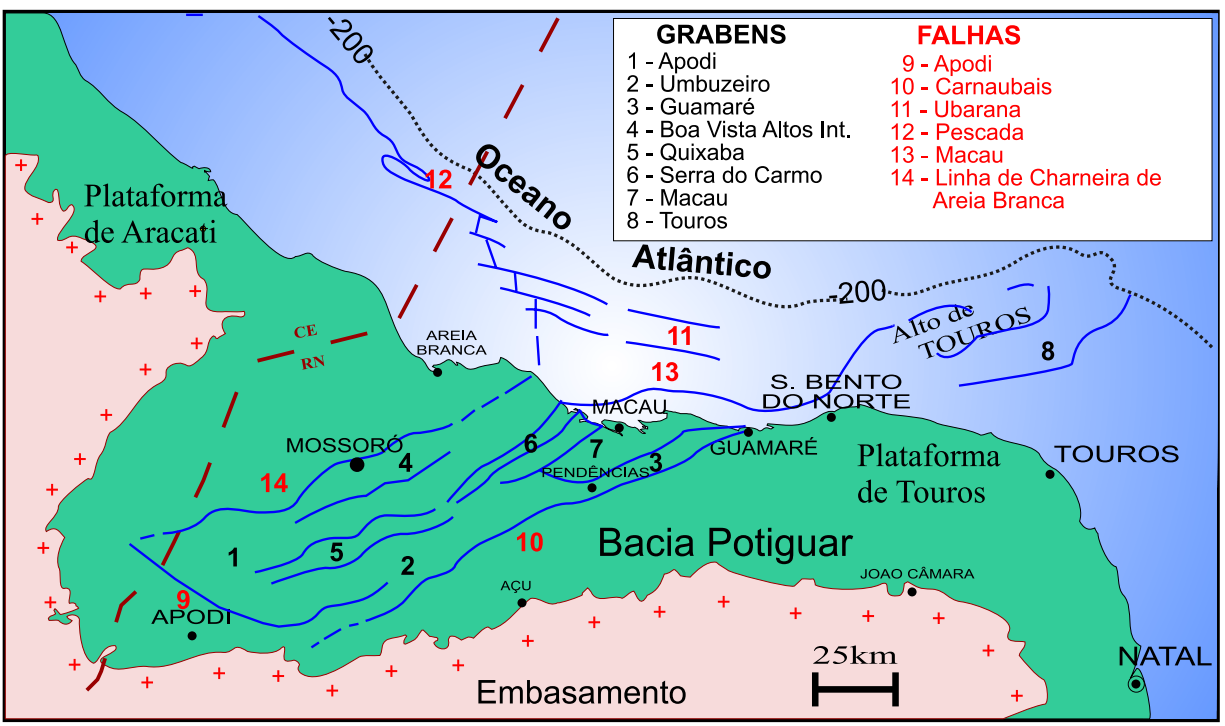

Figura 2 - Arcabouço estrutural da Bacia Potiguar (RN). Modificado de Bertani et al. (1990)

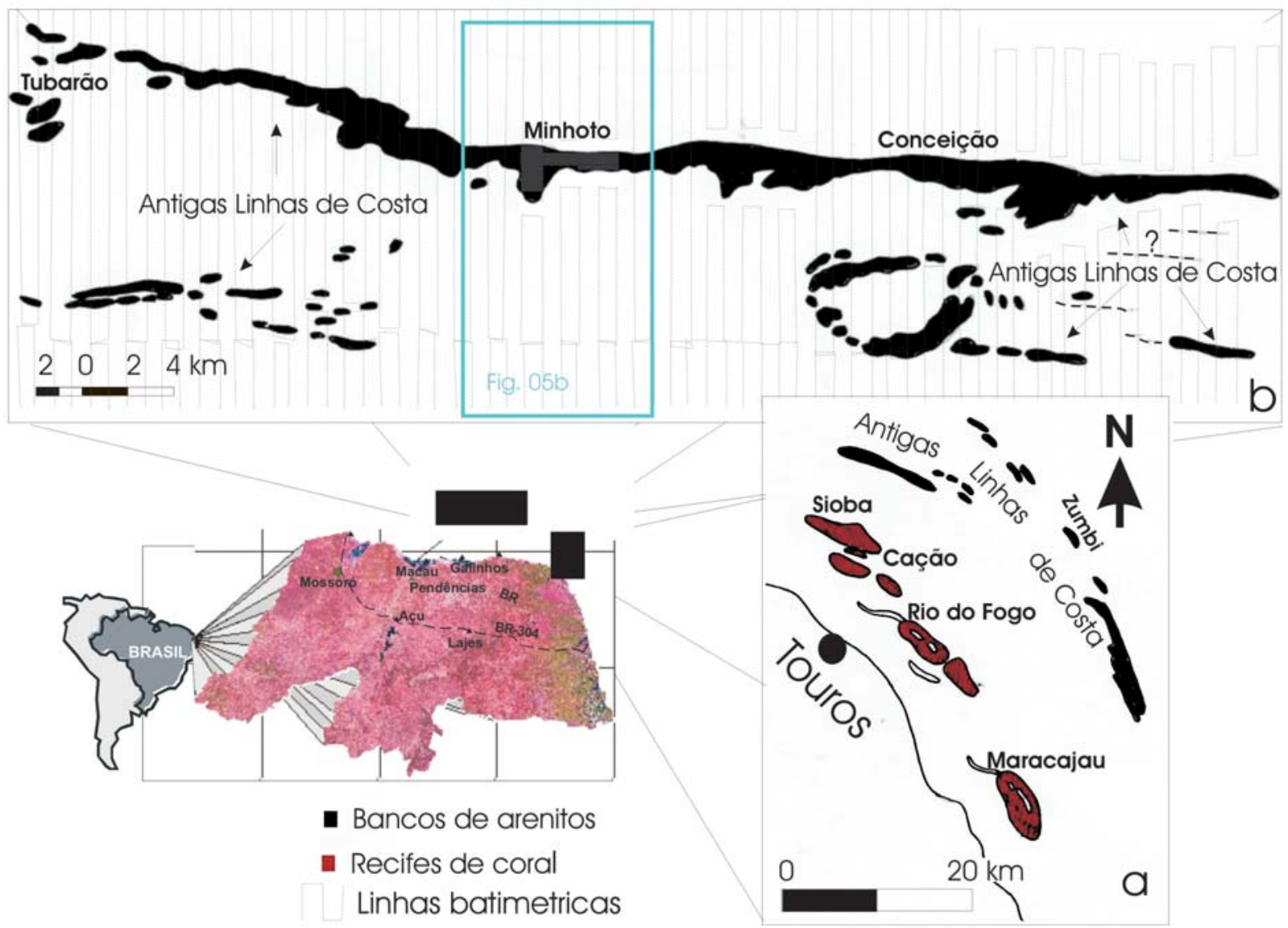

Figura 3 - Mapa das construções carbonáticas, inorgânicas e recifais presentes na região de Touros-RN (a) e entre São Bento e Macau-RN (b). As construções recifais estão destacadas na cor rosa. 
cifes geralmente apresentam orientação NW-SE, paralelos à linha de costa e apresentam-se como conjuntos de cômoros (knol/) e de canteiros (patch reefs). São parcialmente emersos durante a maré baixa, com extensão variando de 8 a $12 \mathrm{~km}$. Segundo De acordo com Amaral (2002), a razão comprimento versus largura destes corpos é de 3/1 a 4/1. Os corais tipo knol/ tem cerca de $0,5 \mathrm{~m}$ de altura, enquanto os tipo patch podem atingir até $6 \mathrm{~m}$. Estão restritos à zona sublitoral túrbida definida por Testa (1997) e Testa \& Bosence (1998). A zona túrbida pode ser observada nas composições coloridas em 4R3G2B e 3R2G1B (Fig. 4a, b).

Em torno de $25 \mathrm{~m}$ de profundidade na borda da plataforma interna, observa-se a presença uma faixa de bancos areníticos constituídos predominantemente por quartzo com cimento carbonático, apresentando também orientação NW-SE. Estes bancos são melhor visualizados na imagem de satélite com a composição colorida em 3R2G1B (Fig. 4b), tendo sido correlacionados por Vianna et al. (1993) a beachrocks análogos aos observados atualmente nas praias da costa adjacente. Testa \& Bosence (1998), por sua vez, os denominam de sedimentos litificados, e os descrevem como afloramentos submersos de arenitos cimentados por carbonato, horizontalmente laminados e acamadados; localmente encrustados por algas coralináceas e esponjas, e colonizados por Halimeda e outras algas.

Por outro lado, os recifes mapeados no litoral setentrional (Conceição, Minhoto, Tubarão), de São Bento do Norte a Macau (Figs. 1b, 3b, 5) são representados principalmente por construções carbonáticas inorgânicas, onde a ocorrência de colônias de corais é inexpressiva. Laborel (1969) já chamava atenção que nesta região, populações de coral das espécies brasileiras Siderastrea stellata e Favia gravida e do hidrocoral Millepora alcicornis formam agregados monoespecíficos, esparsos e mal desenvolvidos. Estas construções são bem ressaltadas na composição colorida em RBG PC1-PC2-PC3 (Fig. 5a). Apresentam forma alongada com orientação predominante aproximadamente $\mathrm{E}-\mathrm{W}$, paralelos à linha de costa, parcialmente emersos durante a maré baixa, com extensão da ordem de dezenas de $\mathrm{km}$. 0 correm a profundidades entre 20 a $30 \mathrm{~m}$ (plataforma interna a média, Fig. 5b) com altura entre 5 a $13 \mathrm{~m}$ e a uma distância em torno de $20 \mathrm{~km}$ da costa. Desta forma, são classificados como bancos (quartzo-areníticos cimentados por carbonato de cálcio). Este mapeamento mostrou que os bancos de Conceição, Minhoto e Tubarão (denominados de Urca pelos pescadores e nas cartas náuticas da DHN), na realidade constituem um único banco, representando uma antiga linha de costa (Figs. 3b, 5a). Outros recifes semelhantes, embora de menor extensão e menor altura, foram mapeados a profundidades em torno de $10 \mathrm{~m}$.
Duas subáreas representativas dos recifes e de construções carbonáticas inorgânicas que são aqui apresentadas foram estudadas in situ: Maracajau (recife, Fig. 4) e Minhoto (banco arenítico Fig. 5).

0 recife de Maracajau (litoral Oriental, Fig. 4) é o maior recife da APA dos Recifes de Corais com 9 km de extensão e 3 km de largura. 0 topo permanece a uma profundidade média inferior a $5 \mathrm{~m}$ e durante as marés baixas fica parcialmente exposto. É constituído por um agrupamento de edificações biogênicas (cômoros e canteiros) de tamanho variável, superfície irregular, permeadas de reentrâncias. Foram construídos pela acumulação de algas calcárias (coralináceas incrustantes) e corais zooxantelados. Corredores de largura e extensão diversos, à moda de labirintos e "piscinas", delimitam essas estruturas concentrando sedimentos terrígenos e fragmentos de carapaças de organismos (Fig. 4c). 0 sedimento de fundo varia de acordo com a energia hidráulica predominante entre areias siliciclásticas e argilas com matéria orgânica. Observa-se um aumento na profundidade e uma diminuição no tamanho dos cômoros e canteiros de oeste para leste. De acordo com a classificação usada neste estudo 0 complexo enquadra-se na categoria de recifes afastados da costa.

Essa morfologia é condicionada pelo povoamento de organismos bentônicos e o modo de crescimento das formas de vida - corais escleractínios e algas coralináceas - responsáveis pela edificação recifal sobre um substrato que tem participação na morfologia dessa edificação (Fig. 4d). Foram registradas as seguintes espécies de corais: Siderastrea stellata (Verrill, 1868), Agaricia fragilis (Dana, 1846), Agaricia agaricites (Verrill, 1901), Porites astreoides (Lamark, 1816), Porites branneri (Rathbun, 1888), Favia gravida (Verrill, 1886), Meandrina brasiliensis (Milne Edwards \& Haime, 1848), Mussimilia hartii (Verrill, 1868). E de hidróides calcários: Millepora alcicornis (Linné, 1758) e Millepora brasiliensis (Verrill, 1868). Na face oceânica do recife (leste), verifica-se a ocorrência de rodólitos arredondados, anêmonas e esponjas.

0 banco do Minhoto (Litoral Setentrional, Fig. 5) apresenta características bastante diferentes daquelas exibidas pelo recife de Maracajau, tanto na topografia e forma quanto no povoamento por parte de organismos bentônicos. A incidência de colônias de corais é praticamente inexistente. 0 banco encontra-se revestido por uma fina camada de algas coralináceas com grande concentração de rodólitos e ocorrências de esponjas também em grande quantidade (Fig. 5c), além de equinodermas, moluscos bivalves, gastrópodes e poliquetas e algas clorofíceas. As algas coralináceas crescem incrustando as superfícies do arenito e fragmentos de conchas ou agregados. Nas áreas de mergulho 0 

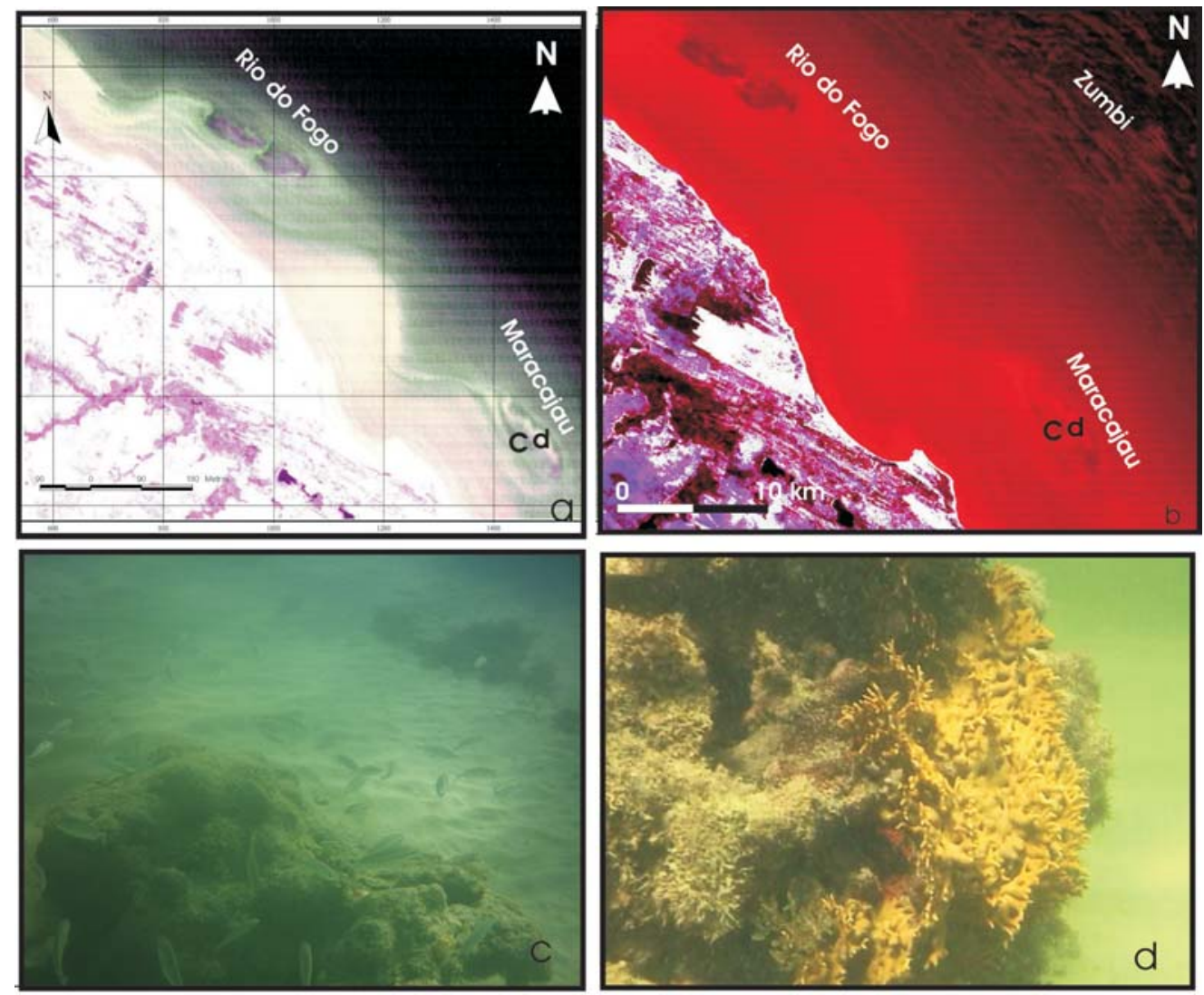

Figura 4 - Construções carbonáticas na região de Touros-RN. (a) Composição colorida em RGB 4-3-2 da Landsat 5-TM de 02 de agosto 1998 (condições de maré de baixamar) e (b) RGB 3-2-1 ressaltando os recifes e os bancos inorgânicos respectivamente. (c) Sedimentos de fundo; (d) Millepora alcicornis nos recifes de Maracajau.

sedimento é de composição bioclástica (teor de carbonato entre 71 e $86 \%)$, constituídos de fragmentos de carapaças de organismos. Aqui, o processo de estabilização e edificação incipiente fica atribuído à ação de algas coralináceas, já que a ocorrência de corais nesse recife se restringiu a uma única colônia de $F$ via gravida já morta. Algas clorofíceas afiguram-se como armadilhas sedimentares, oferecendo proteção ao sedimento contra a ação hidrodinâmica das correntes marinhas. Esses aspectos sugerem diferentes estágios da dinâmica populacional entre as duas estruturas.

Este recife ocorre à mesma profundidade que 0 banco inorgânico identificado na região de Touros (Risca do Zumbi, em torno de $25 \mathrm{~m}$ ).

Análises de lâminas delgadas indicam que 0 banco é constituído por arenito quartzoso de cimento carbonático com características semelhantes aos beachrocks estudados por Caldas (2002) e Stattegger et al. (2006) na área emersa. Entretanto, a unidade litoestratigráfica correspondente aos bancos submersos ainda não foi definida, pois os estudos desenvolvidos até 0 momento não são conclusivos.

\section{CONCLUSÕES}

0 uso do sensoriamento remoto integrado a dados de campo se constitui em um método acessível, rápido e preciso para o mapeamento de áreas submersas onde as águas são límpidas.

Este estudo mostrou que para toda a área estudada ocorrem construções carbonáticas afastadas da costa, sejam elas recifes ou bancos areníticos. Entretanto os recifes de corais ocorrem predominantemente no litoral Oriental (Região de Touros), ao contrário do litoral setentrional (entre São Bento do Norte e Macau) onde as construções carbonáticas são bancos areníticos e a cobertura de carbonato orgânico e de corais é inexpressiva.

Os recifes do litoral oriental são classificados como cômoros (knol/) e canteiros (patch), enquanto no litoral setentrional as construções têm dimensão de bancos.

0 fato das construções inorgânicas se encontrarem a uma 

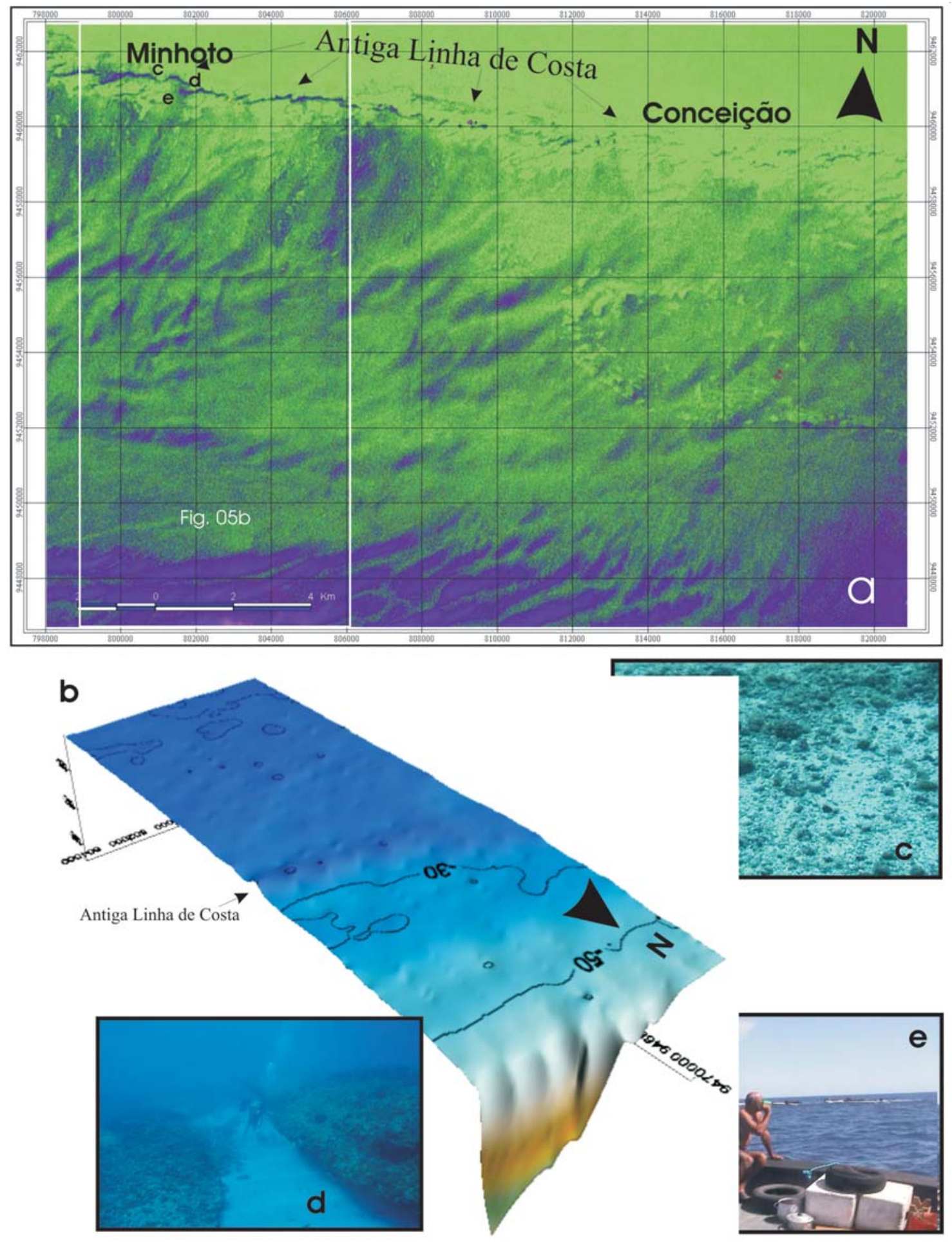

Figura 5 - Banco de arenito do Minhoto-RN. (a) Composição colorida em RGB PC1-PC2-PC3 da Landsat 7-ETM+ de 12 de junho 2000 (condições de maré de baixamar), ressaltando a continuidade dos recifes; (b) modelo da área do Minhoto entre a isóbata de $10 \mathrm{~m}$ e a quebra da plataforma; as isóbatas de 10, 30 e 50 m estão plotadas para controle; (c) campo de rodólitos na parte plana em torno do banco carbonático; (d) vista da parte interna do banco; (e) visão de partes expostas do banco em marés muito baixas. 
profundidade média de $25 \mathrm{~m}$ nas duas áreas estudadas (oriental e setentrional) e apresentarem características semelhantes nos levam a crer que os mesmos são resquícios de uma antiga linha de costa. Entretanto, a unidade litoestratigráfica correspondente a eles não foi definida, podendo a mesma estar relacionada a rochas praiais pleistocênicas ou outros estratos da Bacia Potiguar de idade terciária ou cretácea. A possibilidade de subsidência no litoral setentrional, na região entre a linha do banco e 0 litoral, isolando esta antiga linha de costa, não deve ser descartada.

0 mapeamento das construções carbonáticas desenvolvido neste trabalho se constitui em uma contribuição ao levantamento de dados sobre ocorrência de corais na costa do RN, especialmente em áreas com edificações recifais, bem como às suas condições de desenvolvimento. 0 melhor conhecimento das condições de edificação dessas estruturas submersas, certamente irá contribuir com novos dados inerentes às variações eustáticas na costa do RN.

\section{AGRADECIMENTOS}

Esta pesquisa foi financiada com recursos de Grant $P Q$ (CNPq, Proc. No 3508811999-5) e Projetos PETRORISCO (REDE05/ FINEP/CTPETRO/PETROBRAS/CNPq), CNPq (Proc. No 500407/ 2004-5) e MAR-RN (FINEP/CTINFRA). Os autores agradecem ainda a UFRN (DG, PPGG, CCET) pela disponibilização da infraestrutura necessária ao desenvolvimento da pesquisa, ao CNPq pela concessão de bolsa produtividade de pesquisa aos $2^{\circ}$ e $4^{\circ}$ autores e aos revisores anônimos, que colaboraram para melhorar a qualidade deste artigo. Instituição participante do Programa de Geologia e Geofísica Marinha - PGGM.

\section{REFERÊNCIAS}

ALMEIDA FFM, HASUI Y, BRITO NEVES BB \& FUCK RA. 1977. Províncias estruturais brasileiras. Anais $2^{\circ}$ Simpósio de Geologia do Nordeste, Campina Grande, PB, 242-258.

AMARAL RF. 2002. Mapeamento da Área de Proteção Ambiental dos Recifes de Corais - Fase Exploratória. UFRN, Departamento de Geologia/IDEMA, SUGERCO. Relatório Interno. Ilustrado. Natal. 50 p.

ARARIPE PT \& FEIJÓ F. 1994. Bacia Potiguar. Boletim de Geociências da PETROBRAS, 8(1): 127-141.

ARAÚJO TMF, LEÃO ZMAN \& LIMA OAL. 1984. Evolução do recife de coral da ilha de Itaparica determinada a partir de dados geológicos e geofísicos. Anais do $33^{\circ}$ Congr. Bras. de Geologia, SBG, Rio de Janeiro, RJ, 159-169.

BARRETO AMF, BEZERRA FHR, SUGUIO K, TATUMI SH, YEE M, PAIVA RP \& MUNITA CS. 2002. Late Pleistocene Marine Terrace Deposits in Northeastern Brazil: Sea-Level Changes and Tectonic Implications: Palaeogeography, Palaeoclimatology, Palaeoecology, 179: 57-69.

BERTANI RT, NETO AFA \& MATOS RMD. 1987. O habitat do petróleo da Bacia Potiguar Emersa. Boletim de Geociências da Petrobras, 1(1): 41-49.

BERTANI RT, COSTA IG \& MATOS RMD. 1990. Evolução tectonosedimentar estilo estrutural e habitat do petróleo da Bacia Potiguar. In: RAJA GABAGLIA GP \& MILANI EJ (Ed.). Origem e evolução de bacias sedimentares. Rio de Janeiro, PETROBRAS, p. 291-301.

BRANNER JC. 1904. The Stone Reefs of Brazil, their Geological and Geographical Relations, with a Chapter on the Coral Reefs. Bulletin of the Museum of Comparative Zoology at Harvard College. Geological Series, vol. VII, $275 \mathrm{p}$.

CALDAS LHO. 2002. Late Quaternary Coastal Evolution of the NE Rio Grande do Norte coast, NE Brazil. PhD. Thesis. Kiel University, Germany. $92 \mathrm{p}$.

CHAPPELL J. 1983. Sea level changes and coral reef growth. In: BARNES DJ (Ed.). Perspectives on Coral Reefs. Manuka, Brian Cluston. $46-55$.

CREMONINI OA, GOULART JPM \& SOARES UM. 1996. O Rifte Potiguar: novos dados e implicações tectônicas. Boletim do $4^{\circ}$ Simpósio sobre 0 Cretáceo do Brasil, São Paulo, 89-93.

DAVIES PJ \& KINSEY DW. 1973. Organic and inorganic factors in recent beach rock formation, Heron Island, Great Barrier Reef. Journal of Sedimentary Petrology, 43: 59-81.

DAVIES PJ \& MONTAGGIONI L. 1985. Reef growth and sea-level change: the environmental signature. Proceedings of the $5^{\circ}$ Int. Coral Reef Symposium, Antenne Muséum EPHE, Tahiti, 3: 477-515.

HARTT CF. 1870. Geology and Physical Geography of Brazil. Huntington, Robert E. Krieger Publishing Company. 620 p.

HOPLEY D. 1986. Corals and reefs as indicators of paleo-sea levels with special reference to the Great Barrier Reef. In: VAN DE PLASSCHE 0. (Ed.). Sea-level research: a manual for the collection and evaluation of data. Norwich, Geo Books, 195-228.

KIKUCHI RKP \& LEÃO ZMAN. 1997. Rocas (Southwestern Equatorial Atlantic, Brazil): an atoll built primarily by coralline algae. In: Proceedings of the $8^{\circ}$ ISRS, Int. Coral Reef Symposium, Panama, 1: 731-736.

KIKUCHI RKP \& LEÃO ZMAN. 1998. The effects of Holocene sea level fluctuation on reef development and coral community structure, Northern Bahia, Brazil. Anais da Academia Brasileira de Ciências, 70: 159-171.

LABOREL J. 1967. Les peuplements de Madréporaires des côtes tropicales du Brésil. Thèse Docteur (Université dAix-Marseille), Marseille, $313 p$.

LABOREL J. 1969. Les peuplements de Madréporaires des côtes tropicales du Brésil. Ann. Univ. D’Abidjan, Serie E (Ecologie), 3: 260 p. 
LEÃO ZMAN. 1994. The coral reefs of southern Bahia. In: HETZEL B \& CASTRO CB (Ed). Corals of Southern Bahia. Rio de Janeiro: Editora Nova Fronteira, 151-159.

LEÃO ZMAN \& LIMA OAL. 1982. Caracterização do substrato de recifes de corais holocênicos a partir de dados de sísmica de refração e de perfuração. Revista Brasileira de Geociências, 12: 531-535.

LEÃO ZMAN, KIKUCHI RKP \& TESTA V. 2003. Corals and coral reefs of Brazil. In: CORTÉS J. (Ed.). Latin American Coral Reefs, Elsevier Science B.V., 1-44.

LIMA WSG \& AMARAL RF. 2001. Mapeamento dos Recifes de Corais de Maracajaú/RN com 0 uso de Fotografias Aéreas de Pequeno Formato. Pesquisas em Geociências, 28(2): 417-425.

MAIDA M \& FERREIRA BP. 2004. Os recifes de coral brasileiros. In: ESKINAZI-LEÇA E, NEUMANN-LEITÃO $S$ \& COSTA MF (Org.). Oceanografia: Um Cenário Tropical. Recife: Ed. Bagaço, p. 617-640.

MATOS RMD. 1992. The northeast brazilian rift system. Tectonics, 11(4): 766-791.

NIMER E. 1989. Climatologia do Brasil. IBGE, Rio de Janeiro, 422 pp.

NOLASCO MC \& LEÃO ZMAN. 1986. The carbonate buildups along the northern coast of the State of Bahia, Brazil. In: RABASSA J. (Ed.). Quaternary of South America and Antarctic Peninsula. Rotterdam, A.A. Balkema. 159-190.

OLIVEIRA MIM, BAGNOLI E, FARIAS CC, NOGUEIRA AMB \& SANTIAGO M. 1990. Considerações sobre a geometria, petrografia, sedimentolo- gia, diagênese e idade dos "beachrocks" do Rio Grande do Norte. $36^{\circ}$ Congresso Brasileiro de Geologia, Natal-RN, 2: 621-634.

ROGER J. 1974. Paléontologie Générale. Paris. Masson et Cie, Éditeurs. $419 \mathrm{pp}$.

STATTEGGER K, CALDAS LHO \& VITAL H. 2006. Holocene Coastal Evolution of the Northern Rio Grande do Norte Coast, Brazil. Journal of Coastal Research, SI 39: 150-155.

TESTA V. 1997. Calcareous algae and corals in the inner shelf of Rio Grande do Norte, NE Brazil. Proceedings of the Eighth International Coral Reef Symposium, 1: 737-742.

TESTA V \& BOSENCE DWJ. 1998. Carbonate-Siliciclastic Sedimentation on High-Energy, Ocean-Facing, Tropical Ramp, NE Brazil. In: WRIGHT VP \& BURCHETTE TP (Ed.). Carbonate Ramps, Geol. Soc. London Spec. Pub., 149: 55-71.

TESTA V \& BOSENCE DWJ. 1999. Physical and Biological Controls on the Formation of Carbonate and Siliciclastic Bedforms on the North-East Brazilian: Shelf. Sedimentology, 46: 279-301.

VIANNA ML, SOLEWICZ R, CABRAL AP \& TESTA V. 1991. Sandstream on the Northeast Brazilian Shelf. Continental Shelf Research, 2: 509524.

VIANNA ML, CABRAL AP \& GHERARDI DFM. 1993. TM-Landsat Imagery Applied to the Study of the Impact for Global Climate Change on a Tropical Coastal Environment during the Last Deglaciation. Journal of Remote Sensing, 14: 2971-2983.

\section{NOTAS SOBRE OS AUTORES}

Claude Luiz de Aguilar Santos. Bacharel em Biologia, Especialização em Educação e Mestre em Geodinâmica e Geofísica pela Universidade Federal do Rio Grande do Norte - UFRN. Desde 1979 encontra-se envolvido em atividades de extensão, pesquisa e ensino na área de Biologia Marinha e Paleontologia junto ao Museu Câmara Cascudo - UFRN, do qual foi Diretor no período de 1988 a 1996.

Helenice Vital. Bacharel em Geologia pela Universidade Federal do Rio Grande do Norte - UFRN e Mestre em Geologia Ambiental e Marinha pela Universidade Federal do Pará - UFPA. Concluiu sua Tese de Doutorado na Christian Albrechts Universität zu Kiel, Alemanha em 1996 e desde então vem desenvolvendo projetos de ensino, pesquisa e extensão na área de Geologia e Geofísica Marinha e Monitoramento Ambiental na Universidade Federal do Rio Grande do Norte. Atualmente é pesquisadora 1B do CNPq e Coordenadora do Programa de Recursos Humanos em Geologia, Geofísica e Informática no Setor Petróleo e Gás na UFRN - PRH 22, financiado pela Agência Nacional do Petróleo.

Venerando Eustáquio Amaro. Bacharel em Geologia pela Universidade Federal do Mato Grosso - UFMT e Mestre em Geologia Econômica pela Universidade de Brasília - UNB. Concluiu sua Tese de Doutorado na Universidade de São Paulo em 1998, incluindo dois anos de doutorado-sanduíche na Université Claude BernardLyon 1, França. Desde 1989 encontra-se envolvido em projetos de ensino e pesquisa na área de Sensoriamento Remoto e Geoprocessamento na Universidade Federal do Rio Grande do Norte - UFRN. Atualmente é Coordenador da Rede PETROMAR, Rede N-NE de Monitoramento Ambiental ao Derramamento de Óleo (Rede 05 FINEP CTPETRO CNPq PETROBRAS).

Ruy Kenji Papa de Kikuchi. Bacharel em Geologia pela Universidade de São Paulo - USP e Mestre em Sedimentologia Marinha pela Universidade Federal da Bahia - UFBA. Concluiu sua Tese de Doutorado na área de Evolução dos recifes e comunidades de corais em 2000 na UFBA, onde encontra-se desde então envolvido em atividades de ensino, pesquisa e ensino. É pesquisador CNPq desde 2001. 